\title{
Structural Change in Dimyristoyl Phosphatidyl Choline (DMPC) Molecule Alkyl Chains on the Addition of Cholesterol to DMPC Monolayer: Specific Addition Effect of Cholesterol
}

\author{
Daiki Ito1, Takanari Ikeda1, Keijiro Taga1, Yasushi Yamamoto1* ${ }^{*}$, Zameer Shervani²\#, \\ Masato Yamamoto ${ }^{3}$ \\ ${ }^{1}$ Department of Life Science and Applied Chemistry, Graduate School of Engineering, Nagoya Institute of Technology, \\ Nagoya, Aichi, Japan \\ ${ }^{2}$ Nanomaterials Production Division, Food and Energy Security Research and Product Center, Sendai, Japan \\ ${ }^{3}$ Department of Chemistry, School of Arts and Sciences, Showa University, Kamiyoshida Fujiyoshida, Yamanashi, Japan \\ Email: *yamamoto.yasushi@nitech.ac.jp, " shervani.nanotek@gmail.com
}

How to cite this paper: Ito, D., Ikeda, T., Taga, K., Yamamoto, Y., Shervani, Z. and Yamamoto, M. (2019) Structural Change in Dimyristoyl Phosphatidyl Choline (DMPC) Molecule Alkyl Chains on the Addition of Cholesterol to DMPC Monolayer: Specific Addition Effect of Cholesterol. Journal of Biophysical Chemistry, 10, 15-29.

https://doi.org/10.4236/jbpc.2019.102002

Received: April 19, 2019

Accepted: May 25, 2019

Published: May 28, 2019

Copyright (๑) 2019 by author(s) and Scientific Research Publishing Inc. This work is licensed under the Creative Commons Attribution International License (CC BY 4.0).

http://creativecommons.org/licenses/by/4.0/

\begin{abstract}
We have investigated the morphology of dimyristoyl phosphatidyl choline (DMPC)-cholesterol mixed monolayer formed on the water surface by dropping method using surface tension measurement (STm), Brewster angle microscopy (BAM), and infrared external reflection spectroscopy (IERS). STm results showed negative deviation of the limiting molecular area $\left(A_{0}\right)$ of cholesterol occurred when cholesterol was added to the DMPC monolayer. BAM images showed the expandable DMPC monolayer changed to the condensed rigid monolayer at more than cholesterol mole fraction $\left(x_{C h o l}\right)$ 0.4. IERS recordings showed that the addition of cholesterol at $x_{C h o l}=0.4$ occurred structural change from gauche- to trans- conformation of two DMPC molecule alkyl chains. From these results, it is found that cholesterol molecule has specific properties that cause structural transition of DMPC molecule alkyl chains.
\end{abstract}

\section{Keywords}

Dimyristoyl Phosphatidyl Choline (DMPC), Cholesterol, Surface Tension Measurement (STm), Brewster Angle Microscopy (BAM), Infrared External Reflection Microscopy (IERS), Structural Transition

\section{Introduction}

The monolayers are formed on the water surface when amphiphilic substances 
(surfactant) are two-dimensionally arranged to a monomolecular layer at the air-water interface. Amphiphilic molecules' hydrophobic alkyl chains orient toward the air phase and hydrophilic moieties toward the water subphase. When amphiphilic substance is a lipid, the monolayer corresponds to a half part of the biomembrane. Therefore the lipid monolayer is selected as a biomembrane model to investigate cellular functions such as membrane structure, interaction with functional substances, molecular recognition, and transmission of functional materials in and out of the living biological cell membrane [1] [2] [3] [4].

Cholesterol (Figure 1(a)) is classified under the steroid group. Structurally, it is composed of a steroid skeleton and a hydrophilic hydroxyl group (-OH). Cholesterol is one of the main components in biomembrane and the important organic compound from the viewpoint of various biological phenomena such as controlling of biomembrane conformation, functioning of integral biomembrane proteins, and metabolism in the living body. In these phenomena, "condensation effect" of cholesterol is widely known. The addition of cholesterol in biomembrane alters the order of biomembrane structure and affects the fluidity of the biomembrane. This also promotes thermal and structural stabilities of biomembrane in a wide temperature range. Many studies on the condensation effect have been performed using phospholipid-cholesterol mixtures in monolayer and bilayer systems, and liposomes [2] [5]-[11]. The techniques of thermal analysis, ATR-FTIR and NMR spectroscopy, and molecular simulation [5] [12] [13] [14] [15] [16] showed that the structure of DMPC molecule exchanged from liquid-disordered $\left(L_{\mathrm{d}}\right)$ phase to liquid-ordered $\left(L_{\mathrm{o}}\right)$ phase with an increase in cholesterol mole fraction.

In this communication, we have reported the cholesterol-mixed effect on the morphology of expandable dimyristoyl phosphatidylcholine (DMPC) (Figure 1(b)) monolayer formed on the water surface for various cholesterol mole fractions by dropping method. To this end, physicochemical methods: surface tension measurement (STm), Brewster angle microscopy (BAM), and infrared external reflection spectroscopy (IERS) have been applied. The results have been interpreted to work out an addition effect of cholesterol in the structural change of DMPC molecules.

\section{Materials and Methods}

\subsection{Materials}

Dimyristoyl phosphatidylcholine (DMPC) and cholesterol (both purities: >99\%) were purchased from Avanti polar lipids Inc. (Alabama, USA) and Sigma-Aldrich Corp. (St. Louis, USA), respectively, and were used without further purification. Chloroform (purity: 99\%) were from Wako Pure Chem. Ind. Ltd. (Osaka, Japan). Purified water with conductance $<0.07 \mu \mathrm{S} / \mathrm{cm}$ was obtained using a Super Water Purifying System (WL-21P; Yamato Scientific Corp. Ltd., Tokyo, Japan). 


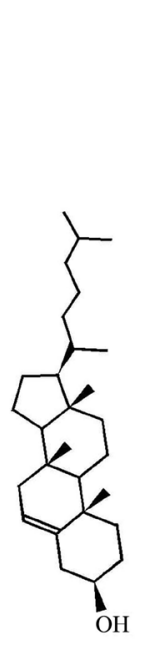

(a)

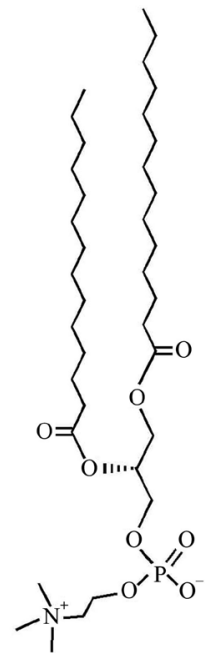

(b)

Figure 1. Structures of cholesterol (a) and DMPC (b).

\subsection{Monolayer Formation}

DMPC and cholesterol were dissolved in chloroform to prepare dropping solution. The solution was dropped on a purified water surface with a $100 \mu \mathrm{l}$ microsyringe (Ge-0583-04; Hamilton Corp., Nevada, USA) to form monolayer. The details of the method of monolayer preparation have been reported in a previously publication [17]. $1 \mu \mathrm{l}$ drop of the solution was dropped gently on the water surface and the next drop was added after $\geq 1 \mathrm{~min}$ so that previous droplet expanded sufficiently on the water surface. The arrival of equilibrium by proper expanding of DMPC and cholesterol on the surface was confirmed by the constant surface tension value attainment after the addition of each droplet (Section 2.3.). The process of the solution addition was repeated until completion of monolayer formation was done. The completion of the monolayer was confirmed by observing the formation of lenses on the water surface and the value of surface tension (Section 2.3.) did not change any more.

\subsection{Surface Tension Measurement (STm)}

Surface tension measurement (STm) for each mixed monolayer was carried out on a Surface Tensiometer (CBVP-A3; Kyowa Interface Science Corp. Ltd., Saitama, Japan) with a platinum Wilhelmy plate. Surface tension was recorded at each dropping at $26^{\circ} \mathrm{C} \pm 1^{\circ} \mathrm{C}$. The surface tension values were then converted into surface pressure $(\pi)$. The $\pi-A$ isotherm curves for each monolayer were constructed as a function of the dropping volume of the monolayer forming components in terms of molecule numbers.

\subsection{Brewster Angle Microscopy (BAM)}

Brewster angle microscopy (BAM) was employed for visualizing the morphologies of each DMPC-cholesterol mixed monolayer using a commercial BAM microscope (EMM633K; Filgen Inc., Nagoya, Japan) attached with an USB-CAP 
type (SD-USB2CUP3; AREA Co. Ltd., Tokyo, Japan) image analysis software. BAM records the monolayer images arise from the refractive index difference between the monolayer and the water phase. BAM device was mounted on a glass dish and p-polarized light of $632.8 \mathrm{~nm}$ wavelength from a $10 \mathrm{~mW} \mathrm{He}-\mathrm{Ne}$ laser was irradiated at the Brewster angle of $53.1^{\circ}$ on each mixed monolayer surface. The reflected light was magnified with a $40 \mathrm{~mm}$ focal length lens and detected by a CCD camera (C5948-70; Hamamatsu Photonics, Hamamatsu). The formation process of each mixed monolayer by dropping method was observed on real-time at $26^{\circ} \mathrm{C} \pm 11^{\circ} \mathrm{C}$. The lateral resolution of $\mathrm{BAM}$ images was about $1 \mu \mathrm{m}$.

\subsection{Infrared External Reflection Spectroscopy (IERS)}

The structure of DMPC molecule alkyl chains in DMPC monolayer and DMPCcholesterol mixed monolayer was investigated by infrared external reflection spectroscopy (IERS). The details of the method of IERS measurement have been reported in a previously publication [18]. An FT-IR spectrometer (FT-IR-600Plus; JASCO Int. Co. Ltd.) with a liquid nitrogen-cooled MCT detector and a reflectance apparatus was employed in combination to make an IERS setup. For every measurement, the solution of DMPC or DMPC-cholesterol (cholesterol mole fraction $\left.\left(x_{C h o l}\right) 0.4\right)$ and chloroform was expanded on the water surface in a poly (tetrafluoroethrene) trough of the size $\left(20 \times 62 \mathrm{~mm}^{2}\right)$ at $26^{\circ} \mathrm{C}^{\circ} \pm 1^{\circ} \mathrm{C}$, according to the dropping method as described above in Section 2.2. The measurements of the above prepared monolayers were conducted at $\pi=35 \mathrm{mN} / \mathrm{m}$. The $p$-polarized IR light at incident angles of $8^{\circ}-20^{\circ}$ and $75^{\circ}$ was irradiated on each monolayer and reflected light was detected by MCT (Mercury-Cadmium-Telluride) detector to investigate the molecular structure and the orientation. More than 1000 scans were averaged with a resolution of $4 \mathrm{~cm}^{-1}$ for the measurements. The IER spectra obtained were converted to the absorption spectra ( $k$-spectra) by the above reported method [18] using Lorentz oscillator model (LOM) calculation under the assumptions, first the $n_{\infty}$ values (the real part of complex refractive index) of the limiting of the high-wavenumber are 1.50 and second the thickness of DMPC or DMPC-Chol monolayer is $2 \mathrm{~nm}$.

\section{Results and Discussion}

\subsection{Surface Tension Measurement (STm)}

Figure 2 shows $\pi$ (surface pressure) versus molecular area $(A),(\pi-A)$ isotherm curves for various DMPC-cholesterol mixed monolayers. The $\pi$ - $A$ isotherms were constructed for a number of cholesterol mole fractions $\left(x_{C h o l}\right): 0.0,0.2,0.3,0.4$, $0.5,0.6,0.8,1.0$ on the water surface using dropping method. The horizontal axis represents $A / \mathrm{nm}^{2}$ calculated from molecular numbers in the dropping volume and the vertical axis represents $\pi / \mathrm{mN} \times \mathrm{m}^{-1}$ recorded after dropping the sample solution on the water surface. The value of $\pi$ was noted finally when $\pi$ reached at a constant value. 


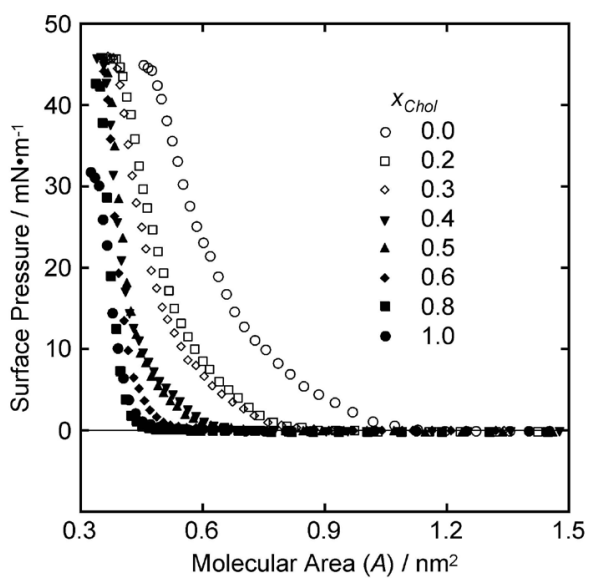

Figure 2. $\pi-A$ isotherm curves of various DMPC-cholesterol mixed monolayers (cholesterol mole fraction $x_{C h o l}=0.0,0.2,0.3,0.4,0.5$, $0.6,0.8,1.0)$ on the water surface at $26^{\circ} \mathrm{C}$, using dropping method.

The DMPC monolayer $\pi$ - $A$ isotherm curve (०) without cholesterol is the same as reported previously [19] [20]. The $\pi$ value increased gradually at about 1.2 $\mathrm{nm}^{2}$ of $A$ and showed a monotonous increase up to $43 \mathrm{mN} / \mathrm{m}$. The shape of the curve was smooth and similar to that obtained by compression method [21] [22] [23]. The limiting molecular $A_{0}$ was $0.74 \pm 0.02 \mathrm{~nm}^{2} /$ molecule corresponded to $40 \mathrm{mN} / \mathrm{m}$ of $\pi$ and the value was very similar $\left(0.73 \mathrm{~nm}^{2} /\right.$ molecule $)$ obtained at 40 $\mathrm{mN} / \mathrm{m}$ in compression method. From the pattern of $\pi-A$ isotherm curve, it was confirmed that DMPC monolayer by dropping method formed liquid expanded (LE) state where two DMPC molecule's alkyl chains were disordered partially. The structure of alkyl chains contained gauche conformation, thereby the crosssectional area would be larger than of the hydrophilic group.

As shown in Figure 2, with an increase of $x_{\text {Chol }}$ from $\square$ to $\boldsymbol{~}$, the curves of DMPC-cholesterol mixed monolayer shifted to the left and approached to a plot pattern of cholesterol monolayer $(\bullet)$. At $>x_{\text {Chol }}=0.4(\boldsymbol{\nabla})$, the curves showed steep rise and approached to that of similar to cholesterol monolayer $(\bullet)$, though each $A$ value where each curve started to rise was different. Each $A_{0}$ for $>X_{C h o l}=$ 0.4 showed values close to that of cholesterol monolayer, confirmed that at $>X_{\text {Chol }}$ $=0.4$ mixed monolayers are in a condensed state on the water surface.

For the cholesterol monolayer, the $\pi$ - $A$ isotherm curve $(\bullet)$ in Figure 2 showed that the $\pi$ increased a little gradually and subsequently sudden steeply up to 30 $\mathrm{mN} / \mathrm{m} . A_{0}$ was $0.39 \pm 0.02 \mathrm{~nm}^{2} /$ molecule at $30 \mathrm{mN} / \mathrm{m}$ of $\pi$ and this value was also very similar $\left(0.40 \mathrm{~nm}^{2} /\right.$ molecule at $\left.35 \mathrm{mN} / \mathrm{m}\right)$ in compression method [22] [23] [24] [25]. The obtained $A_{0}$ was also identical ( $0.41 \mathrm{~nm}^{2} /$ molecule at $\left.34 \mathrm{mN} / \mathrm{m}\right)$ as reported previously by dropping method [11].

$A_{0}$ of DMPC-cholesterol mixed monolayer in this research was compared to that of the ideal mixed monolayer that is calculated from each $A_{0}$ of DMPC and cholesterol monolayers. Figure 3 is $x_{C h o l}$ dependence versus $A_{0}$ plot $(\bullet)$ of DMPC-cholesterol mixed monolayer. The horizontal axis represents $x_{C h o l}$ and the vertical axis is $A_{0} / \mathrm{nm}^{2}$ determined from $\pi$-A isotherm curve (Figure 2). The 


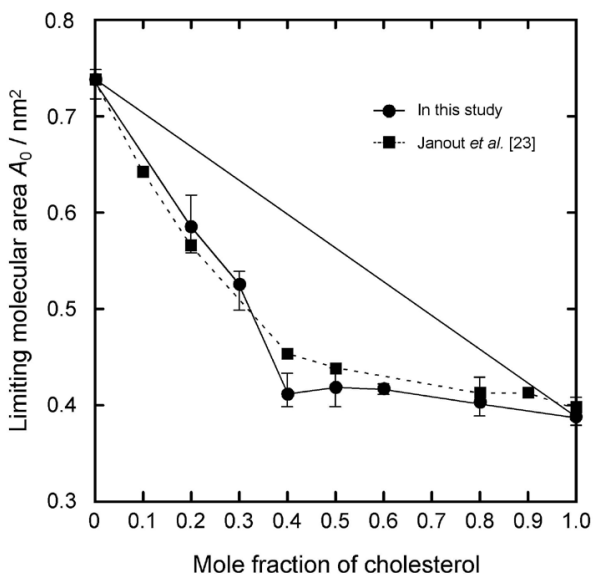

Figure 3. Cholesterol mole fraction dependence of limiting molecular area $A_{0}$. $A_{0}$ in the case without the cholesterol-mixed effect is indicated by straight line.

•: In this study, -: Previously reported data of Janout et al. [23].

straight line (-) is drawn as an ideal additivity on each $x_{C h o l}$ In the figure, previously reported data of $A_{0}(\cdot)$ [23] versus $x_{C h o l}$ obtained by compression method have been compared with our results $(\bullet)$. From the data of our plot $(\bullet)$, the addition of cholesterol in the DMPC monolayer showed a negative deviation from the ideal line. This indicates that cholesterol molecules have a condensation effect on DMPC monolayer at various $x_{C h o l}$ [26] [27]. The tendency of negative deviation in dropping method $(\bullet)$ and compression method (•) was almost same, although the negative deviation of plot $(\bullet)$ was slightly larger than that of plot $(\mathbf{-})$ at more than $x_{C h o l}=0.4$. Therefore the deviation occurred in both the methods suggests that the DMPC-cholesterol mixed monolayer formation by dropping and compression methods are same and thus cholesterol condensation effect of the mixed monolayer is also same in both methods. The result of cholesterol condensation effect on DMPC monolayer was different from that of dipalmitoyl phosphatidyl choline (DPPC) monolayer, where the condensation effect of cholesterol on DPPC monolayer by dropping method was twice larger than compression method [11].

\subsection{Brewster Angle Microscopy (BAM)}

A series of BAM images containing five cholesterol mole fractions $\left(x_{C h o l}=0.0,0.3\right.$, $0.4,0.6,1.0)$ of DMPC-cholesterol mixed monolayers are shown in Figure 4. The $x_{\text {Chol }}$ of Figures 4(a)-(d), Figures 4(e)-(h), Figures 4(i)-(l), Figures 4(m)-(p), and Figures $4(\mathrm{q})-(\mathrm{t})$, are $0.0,0.3,0.4,0.6$, and 1.0, respectively. For each $x_{\text {Chol }}$ value, BAM images were recorded at four $\pi$ values as given in the figure.

For $x_{\text {Chol }}=0.0$ (Figures $4(\mathrm{a})$-(d), the images showed that DMPC molecules form an expanding monolayer. Therefore the whole image was shown in slight bright contrast at $0.5 \mathrm{mN} / \mathrm{m}$ (Figure $4(\mathrm{a})$. With an increase of $\pi$ to 10 and 32 $\mathrm{mN} / \mathrm{m}$ as shown in Figure 4(b) and Figure 4(c), respectively, images became brighter as the DMPC monolayer continued to form homogeneously. At $\pi=42$ $\mathrm{mN} / \mathrm{m}$ in Figure $4(\mathrm{~d})$, the brightness of image increased further and the image 

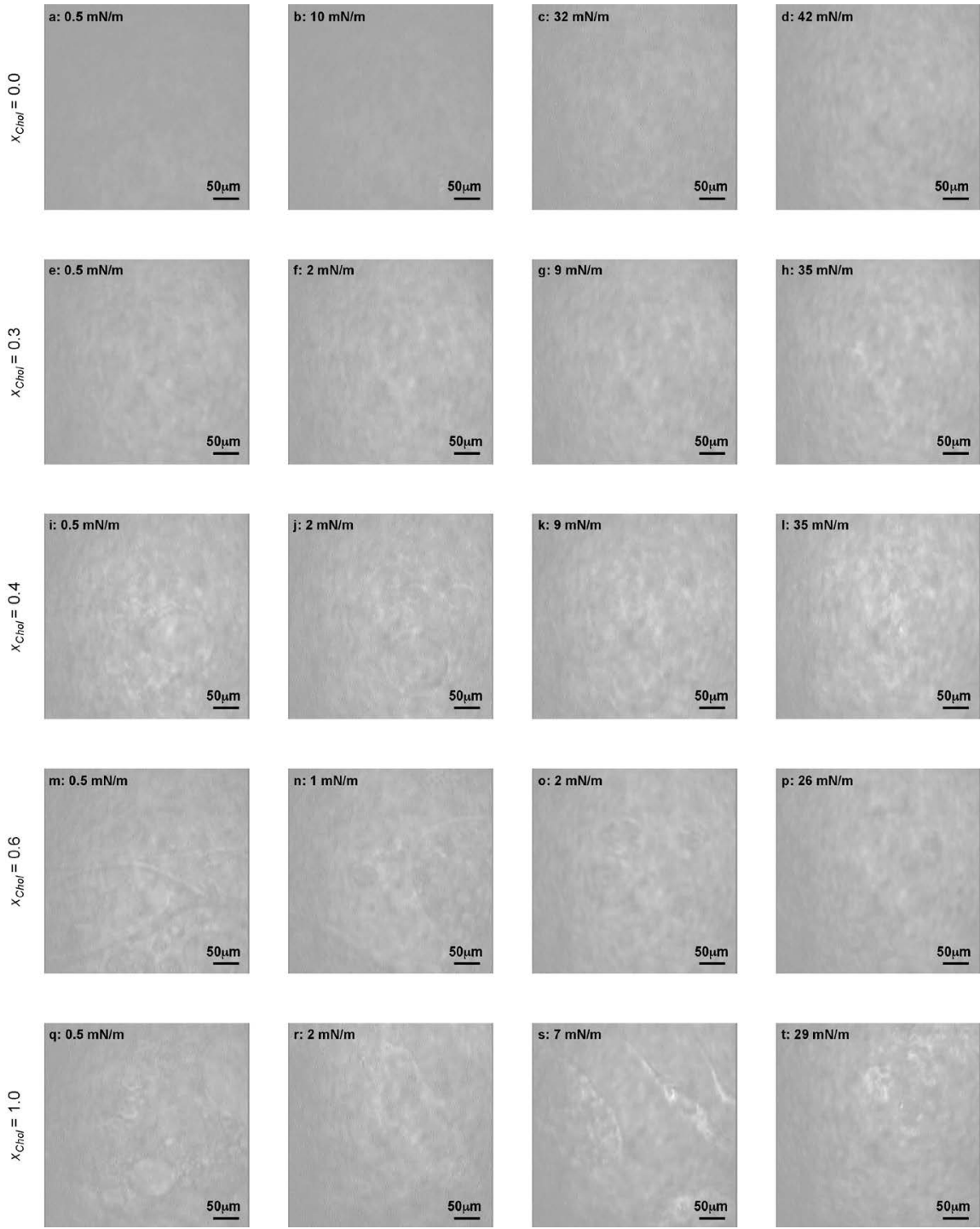

Figure 4. BAM images of DMPC-cholesterol mixed monolayer at various surface pressures. (a)-(d): DMPC monolayer; (e)-(h): Mixed monolayer of $x_{\text {Chol }}=0.3$; (i)-(l): Mixed monolayer of $x_{\text {Chol }}=0.4 ;(\mathrm{m})$-(p): Mixed monolayer of $x_{\text {Chol }}=0.6$; (q)-(t): cholesterol monolayer.

was brightest among all the above three $\pi$ values, thereby confirmed that the completion of monolayer occurred at the water surface. The increase in brightness of the BAM images corresponded to a gradual increase in $\pi$ values as shown in the $\pi$ - $A$ isotherm curve of DMPC monolayer (Figure 2, plot (०)), confirmed the formation of LE state. The monolayer formation process was almost the same as reported previously by compression method [28]. The DMPC of an expandable molecule formed an expanded monolayer by both methods, as the aggregation process on the water surface remained the same in both methods.

On the DMPC-cholesterol mixed monolayer at $x_{\text {Chol }}=0.3$ (Figures $4(\mathrm{e})-(\mathrm{h})$ ), images were similar to the DMPC monolayer (Figures 4(a)-(d)). Comparing images Figure 4(e) with Figure 4(a), the image Figure 4(e) was somewhat 
brighter than Figure 4(a). It is due to that the DMPC molecules were attracted to each other by the addition of cholesterol into the DMPC monolayer. The above change in BAM images corresponded to the addition effect of cholesterol in Figure 2 (plot $\diamond$ ) which shifted to the left by the addition of cholesterol. Similarly the mixed monolayer of $x_{C h o l}=0.2$ also showed similar changes in BAM images as those of $x_{\text {Chol }}=0.3$ (images not shown).

On the DMPC-cholesterol mixed monolayer at $x_{\text {Chol }}=0.4$ (Figures 4(i)-(1)), the images were different from $x_{C h o l}=0.0,0.2$, and 0.3. At quite lower value of $\pi$ $=0.5 \mathrm{mN} / \mathrm{m}$ (Figure 4(i)), the image showed line and circle type domain. The appearance of this domain indicated that condensed aggregates of DMPC and cholesterol molecules formed at the water surface. With an increase of $\pi$ to 2 $\mathrm{mN} / \mathrm{m}$ (Figure $4(\mathrm{j})$ ), the domain became larger and the image was also brighter than Figure 4(i). At $\pi=9 \mathrm{mN} / \mathrm{m}$ (Figure $4(\mathrm{k})$ ), the homogeneous state appeared and the domain obtained at $2 \mathrm{mN} / \mathrm{m}$ disappeared. At higher $\pi=35$ $\mathrm{mN} / \mathrm{m}$ (Figure $4(1)$ ), the image became still brighter and indicating that the completion of the homogeneous DMPC-cholesterol monolayer formation on the water surface. The above change in BAM images were similar to the following cholesterol monolayer images (Figures $4(\mathrm{q})$-(t)) which formed by the cholesteroltype condensed monolayer on the water surface. The change also corresponded to the addition effect of cholesterol in Figure 2 (plot $\mathbf{\nabla}$ ) which shifted to the left by the addition of cholesterol and was similar to the cholesterol-like monolayer shape.

On the DMPC-cholesterol mixed monolayer at $x_{\text {Chol }}=0.6$ (Figures $4(\mathrm{~m})$-(p)), the images were similar to those of $x_{\text {Chol }}=0.4$ (Figures 4(i)-(1)) and also similar to the following cholesterol monolayer images (Figures $4(\mathrm{q})-(\mathrm{t})$ ) which formed by the cholesterol-type condensed monolayer on the water surface, as same as $x_{\text {Chol }}=0.4$ (Figures $4(\mathrm{i})$-(1)). The change in BAM images also corresponded to the addition effect of cholesterol in Figure 2 (plot $\bullet$ ) which shifted to the left by the addition of cholesterol and was similar to the cholesterol-like monolayer shape. Similarly the mixed monolayer of $x_{C h o l}=0.8$ also showed similar changes in BAM images as those of $x_{\text {Chol }}=0.4$ and 0.6 (images not shown).

On the cholesterol monolayer at $x_{C h o l}=1.0$ (Figures $4(\mathrm{q})-(\mathrm{t})$ ), the images showed that cholesterol molecules form a condensed monolayer. At quite lower value of $\pi=0.5 \mathrm{mN} / \mathrm{m}$ (Figure $4(\mathrm{q})$ ), the image showed line and circle type domain, thereby the appearance of condensed aggregates of cholesterol on the water surface. With an increase of $\pi$ to 2 and $7 \mathrm{mN} / \mathrm{m}$ as shown in Figure $4(\mathrm{r})$ and Figure 4(s), respectively, the number and size of line- and circle-type domains appeared at $\pi=0.5 \mathrm{mN} / \mathrm{m}$ increased and enlarged gradually. The images also became brighter due to high condensation forces of cholesterol molecules. At $\pi$ $=29 \mathrm{mN} / \mathrm{m}$ in Figure $4(\mathrm{t})$, the domains appeared at $<7 \mathrm{mN} / \mathrm{m}$ disappeared and the image became homogeneous. Among all images of $x_{C h o l}=1.0$, Figure $4(\mathrm{t})$ was most bright and thus the formation of homogeneous cholesterol monolayer was completed on the water surface. The monolayer formation process of cho- 
lesterol (Figures $4(\mathrm{q})-(\mathrm{t})$ ) was almost the same as those of reported previously by compression method [29] [30] [31]. Therefore for condensable molecule such as cholesterol, the condensed monolayer is formed through the same aggregation process on the water surface either by dropping or compression methods.

\subsection{Infrared External Reflection Spectroscopy (IERS)}

Figure 3 showed the dependency of $A_{0}$ on the added cholesterol in the mixed monolayer. At more than $X_{C h o l}=0.4$, the plot confirmed that $A_{0}$ values of lipid approached to the cholesterol monolayer. It is specific that DMPC molecule reduced its $A_{0}$ by the addition of cholesterol, because two DMPC molecule alkyl chains contain gauche conformation in the monolayer. It is predicted that the change in DMPC molecule alkyl chains conformation occurred by the addition of cholesterol. The IERS measurement has been performed for the investigation of alkyl chain structural conformation change occurred in DMPC molecules of the DMPC-cholesterol mixed monolayer.

Figure 5(a) and Figure 5(b) are IER spectra of DMPC monolayer without addition of cholesterol at $\pi=35 \mathrm{mN} / \mathrm{m}$ as shown in Figure 2 (plot $\circ$ ). Figure 5(a) shows the result measured at the incident angle (IA) of $75^{\circ}$ and Figure 5(b) at IA $10^{\circ}$, respectively. The horizontal axis represents wavenumber $/ \mathrm{cm}^{-1}$ and the vertical axis represents reflectance/\%. The dotted lines (---) show measured spectrum and solid lines (-) are the simulated spectrum. Figure $5(c)$ is the absorption spectra ( $k$-spectra) obtained from simulation calculation based on the solid experimental line in Figure 5(a) and Figure 5(b) [18]. In Figure 5(c), the vertical axis represents imaginary part of the complex refractive index $(k)$, solid line (-) spectra $\left(k_{\mathrm{xy}}\right)$ is of the components parallel to the surface plane (in plane), dotted line (---) spectra $\left(k_{\mathrm{z}}\right)$ is of the components perpendicular to the surface plane (out of plane) [18].

As shown in the $k$-spectra of Figure 5(c) $\left(k_{\mathrm{z}}\right)$, five absorption peaks were assumed in simulation at 2958,2920,2897, 2872 and $2852 \mathrm{~cm}^{-1}$. These peaks were assigned to $\mathrm{CH}_{3}$ anti-symmetric stretching vibration band $v_{\mathrm{as}}\left(\mathrm{CH}_{3}\right), \mathrm{CH}_{2}$ anti-symmetric stretching vibration band $v_{\mathrm{as}}\left(\mathrm{CH}_{2}\right)$, Fermi resonance $\mathrm{CH}_{3}$ symmetric stretching vibration band $v_{\mathrm{s}}\left(\mathrm{CH}_{3}\right)$, and $\mathrm{CH}_{2}$ symmetric stretching vibration band $v_{s}\left(\mathrm{CH}_{2}\right)$, respectively. The two peaks at 2920 and $2852 \mathrm{~cm}^{-1}$ were strong in $k_{\mathrm{xy}}$ spectrum assigned to the methylene groups in the two DMPC molecule alkyl chains.

To investigate the form of the two alkyl chains (the hydrophobic group) in the monolayer, we focused on the information of two stretching vibration bands $\left(v_{\text {as }}\left(\mathrm{CH}_{2}\right)\right.$ and $\left.v_{\mathrm{s}}\left(\mathrm{CH}_{2}\right)\right)$ of methylene groups $\left(-\mathrm{CH}_{2}-\right)$ in Figure $5(\mathrm{c})$. The bands $\left(v_{\text {as }}\left(\mathrm{CH}_{2}\right)\right.$ and $\left.v_{\mathrm{s}}\left(\mathrm{CH}_{2}\right)\right)$ appeared at 2920 and $2852 \mathrm{~cm}^{-1}$, respectively are characteristics of gauche conformation of alkyl chain [32] [33]. Therefore the hydrophobic DMPC molecule alkyl chains in the monolayer are in an irregularly kinked form.

Figure 6(a) shows IER spectra of DMPC-cholesterol $\left(x_{\text {Chol }}=0.4\right)$ mixed mo- 
nolayer of $\pi=35 \mathrm{mN} / \mathrm{m}$ at the IA of $8^{\circ}$. The dotted lines (---) show measured spectrum and solid lines (-) are the simulated spectrum. In Figure 6(b), the solid line (-) shows the absorption spectrum ( $k$-spectrum) obtained from the simulation calculation [18] conducted using the solid line (-), the experimental IER recording shown in Figure 6(a). The solid lines (-) of Figure 6(b) is the absorption spectrum ( $k$-spectra) $\left(k_{\mathrm{xy}}\right)$ obtained from simulation calculation based on the solid experimental line in Figure 6(a) [18]. For a comparison, the $\left(k_{x y}\right)$ of DMPC monolayer in Figure 5(c) is also described as the dotted lines (---) in Figure 6(b).

The two strong absorption peaks $v_{\mathrm{as}}\left(\mathrm{CH}_{2}\right)$ and $v_{\mathrm{s}}\left(\mathrm{CH}_{2}\right)$ were optimized to fit the experimental IER spectrum, resulting the peak position of $k_{\mathrm{xy}}$ (solid lines $(-)$ ) at $2919 \mathrm{~cm}^{-1}$ and $2849 \mathrm{~cm}^{-1}$, respectively, for DMPC-cholesterol $\left(x_{C h o l}=0.4\right)$ mixed monolayer. These peaks were assigned to $\mathrm{CH}_{2}$ anti-symmetric stretching vibration band $v_{\mathrm{as}}\left(\mathrm{CH}_{2}\right)$ and $\mathrm{CH}_{2}$ symmetric stretching vibration band $v_{\mathrm{s}}\left(\mathrm{CH}_{2}\right)$, respectively.

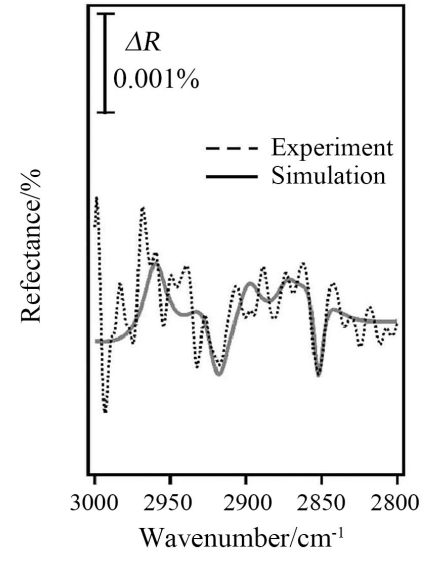

(a)

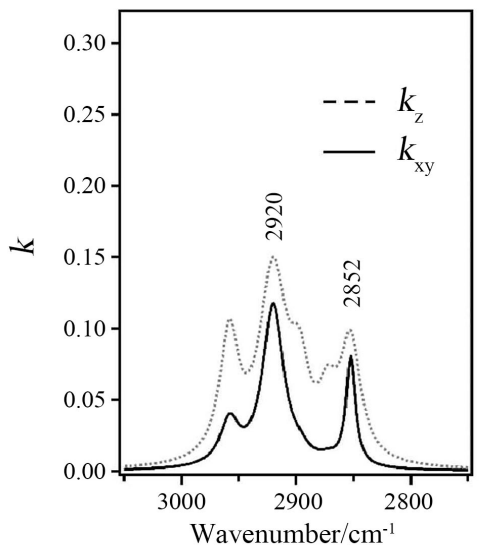

(c)

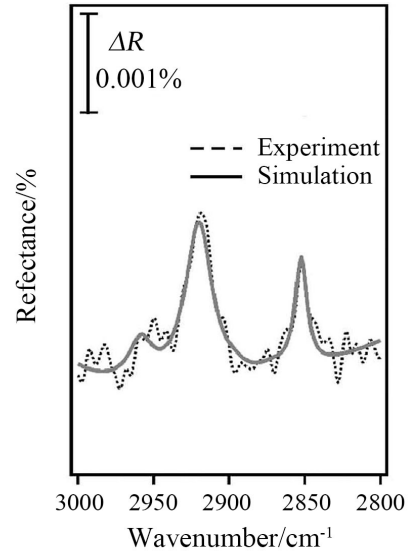

(b)

Figure 5. (a) and (b): IER spectra of DMPC monolayer at around a pressure of $35 \mathrm{mN} / \mathrm{m}$. Incident angle is $75^{\circ}$ (a) and $10^{\circ}$ (b), respectively. Dotted-line is measured spectrum and solid-line is simulated spectrum. (c): absorption spectra ( $k$-spectra) provided from simulated IER spectra of (a) and (b). Solid-line is in plane $\left(k_{x y}\right)$ and dotted-line is out of plane $\left(k_{z}\right)$. 


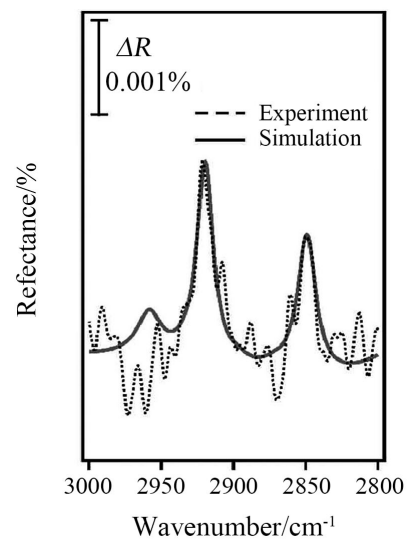

(a)

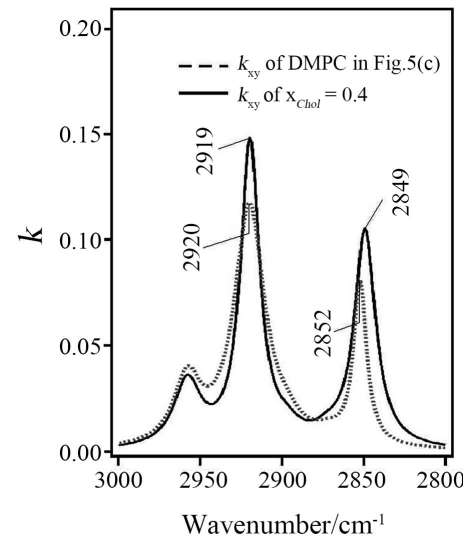

(b)

Figure 6. (a): IER spectra of DMPC-cholesterol mixed monolayer $\left(x_{C h o l}=0.4\right)$ at around a pressure of $35 \mathrm{mN} / \mathrm{m}$. Incident angle is $10^{\circ}$. Dotted-line is measured spectrum and solid-line is simulated spectrum. (b): absorption spectra ( $k$-spectra in plane) provided from the simulation. Solid-line is mixed monolayer and dotted-line is DMPC monolayer $k_{\mathrm{xy}}$ in Figure $5(\mathrm{c})$.

Comparing the two $k$-spectra of DMPC monolayer with (-) and without cholesterol (---) in Figure 6(b), two differences in peak position and absorption intensity were noticed. The peak positions of DMPC-cholesterol spectrum (-) $v_{\text {as }}\left(\mathrm{CH}_{2}\right)$ and $v_{\mathrm{s}}\left(\mathrm{CH}_{2}\right)$ at 2919 and $2849 \mathrm{~cm}^{-1}$, respectively, were at shorter wavelengths than DMPC spectrum (---) $v_{\mathrm{as}}\left(\mathrm{CH}_{2}\right)$ and $v_{\mathrm{s}}\left(\mathrm{CH}_{2}\right)$ at 2920 and $2852 \mathrm{~cm}^{-1}$, respectively. The absorbance intensities of the former mixed monolayer's bands were 1.3 times higher than the latter.

Snyder and Strauss [32] reported in C-C bond of polymethylene a lower (blue shift) in two $v_{\text {as }}\left(\mathrm{CH}_{2}\right)$ and $v_{\mathrm{s}}\left(\mathrm{CH}_{2}\right)$ stretching vibration bands resulted due to change from gauche- to trans- conformation. Therefore the blue shift is suggested that the kinked form (gauche conformation) of DMPC molecules decreased by the addition of cholesterol to the monolayer. Thus addition of cholesterol caused the structural transition from gauche- to trans- conformation. The increase in the absorption intensity of the two peaks $\left(v_{\mathrm{as}}\left(\mathrm{CH}_{2}\right)\right.$ and $\left.v_{\mathrm{s}}\left(\mathrm{CH}_{2}\right)\right)$ by the addition of cholesterol also supports the structural change described above. The trans conformation consecutively arranged in the alkyl chain in all-trans zig-zag form with the long axis along the surface normal with parallel to the $-\mathrm{CH}_{2}$-plane at the surface plane. This arrangement makes the peak intensities of $v_{\text {as }}\left(\mathrm{CH}_{2}\right)$ and $v_{\mathrm{s}}\left(\mathrm{CH}_{2}\right)$ higher (Figure $6(\mathrm{~b})$ ) on the addition of cholesterol in the $k$-spectra (in $k_{\mathrm{xy}}$ plane) [18].

The blue shifted stretching frequency of $v_{\text {as }}\left(\mathrm{CH}_{2}\right)$ and $v_{s}\left(\mathrm{CH}_{2}\right)$ vibration bands of the two DMPC molecule alkyl hydrophobic chains, with the addition of cholesterol in the DMPC monolayer, leads the mixed monolayer more ordered and condensed by a decrease in the area of those alkyl chains with the structural change from disorder to order. This also corresponds to the negative deviation from the ideal line in Figure 3. The structural transition from gauche- to transconformation due to the addition of cholesterol occurred in the DMPC molecule 
alkyl chains. The alkyl chains ordered structure and more condensed state also corresponded to the STm result in Figure 2 that the shape of $\pi$ - $A$ isotherms curve showed LC state close to that of cholesterol.

\section{Conclusions}

In this research, we have investigated the cholesterol addition effect on the DMPC monolayer formed by dropping method. The $\pi-A$ isotherm curves constructed by STm on various DMPC-cholesterol mixed monolayer showed the addition effect of cholesterol on the expandable DMPC monolayer, as a negative deviation of $A_{0}$ from the ideal mixed $A_{0}$. When the amount of cholesterol was $>x_{C h o l}=$ $0.4 A_{0}$ was almost the same as cholesterol. The recorded BAM images also supported STm results, the images containing cholesterol at $<x_{\text {Chol }}=0.4$ showed expandable state and at $>x_{\text {Chol }}=0.4$ condensed state similar to the cholesterol. Moreover IER spectra showed the structural difference of two DMPC molecule alkyl chains between $x_{C h o l}=0.0$ and 0.4 , therefore the alkyl chains were in the gauche (kinked) form at $x_{\text {Chol }}=0.0$ and the conformation changed to trans (straight) form at $x_{\text {Chol }}=0.4$.

The results obtained in this research showed first the ordering of DMPC molecule occurred even at the level of monolayer on the water surface and the ordering was related not only to the general condensation effect of cholesterol but also to the gauche- to trans- conformation change in the two DMPC molecule alkyl chains. The ordering might have induced changes in the density and orientation of hydrophilic group of DMPC molecule at monolayer-water interface. More experiments are needed to obtain further information about the effect of cholesterol on the DMPC molecules in the DMPC-cholesterol mixed monolayer, using several other physicochemical methods such as surface potential measurement and vibration sum frequency generation (VFSG) spectroscopy.

\section{Acknowledgements}

This work was supported by a Grant-in-Aid from the Ministry of Science, Education, Sports and Culture (No. 21750143 and 24550154).

\section{Conflicts of Interest}

The authors declare no conflicts of interest regarding the publication of this paper.

\section{References}

[1] Shimabayashi, S., Terada, H. and Okabayashi, H. (1990) Biocolloid 1 \& 2. Hirokawa Publishing, Tokyo.

[2] Gennis, R.G. (1990) Biomembrane, Molecular Structure and Function. Springer, New York

[3] Kawai, T. (2005) Interfaces and Surfactants-Fundamentals and Applications. Japan Oil Chemists' Society, Tokyo.

[4] Suezaki, Y. (2007) Physics of lipid membrane. Kyushu University Press, Fukuoka. 
[5] Mabrey, S., Mateo, P.L. and Sturtevant, J.M. (1978) High-Sensitivity Scanning Calorimetric Study of Mixtures of Cholesterol with Dimyristoyl- and Dipalmitoyl-Phosphatidylcholine. Biochemistry, 17, 2464-2468.

https://doi.org/10.1021/bi00605a034

[6] Finegold, L. (1993) Cholesterol and Membrane Models. CRC Press, Inc., Boca Raton, FL.

[7] Takahashi, H., Yasue, T., Ohki, K. and Hatta, I. (1996) Structure and Phase Behaviour of Dimyristoylphosphatidic Acid/Poly(L-Lysine) Systems. Molecular Membrane Biology, 13, 233-240. https://doi.org/10.3109/09687689609160601

[8] McMullen, T.P.W. and McElhaney, R.N. (1996) Physical Studies of Cholesterol-Phospholipid Interactions. Current Opinion in Colloid Interface Science, 1, 83-90. https://doi.org/10.1016/S1359-0294(96)80048-3

[9] Hatta, I. and Murata, M. (2000) Dynamics of Biomembrane. Kyoritsu Shuppan Co., Tokyo.

[10] Quinn, P.J. and Wolf, C. (2009) The Liquid-Ordered Phase in Membranes. Biochimica Biophysica Acta, 1788, 33-46. https://doi.org/10.1016/j.bbamem.2008.08.005

[11] Yokoyama, T., Yoshida, D., Mori, H., Okabe, M., Shervani, Z., Taga, K., Yamamoto, Y., Sumino, A., Dewa, T., Nango, M. and Yamamoto, M. (2016) Morphological Observation of Specific Condensation Effect of Cholesterol on Dipalmitoyl Phosphatidyl Choline (DPPC) Monolayer by Dropping Method. Journal of Biophysical Chemistry, 7, 98-109. https://doi.org/10.4236/jbpc.2016.74009

[12] Almeida, P.F.F., Vaz, W.L.C. and Thompson, T.E. (1992) Lateral Diffusion in the Liquid Phases of Dimyristoylphosphatidylcholine/Cholesterol Lipid Bilayers: A Free Volume Analysis. Biochemistry, 31, 6739-6747. https://doi.org/10.1021/bi00144a013

[13] Trandum, C., Westh, P., Jorgensen, K. and Mouritsen, O.G. (2000) A Thermodynamic Study of the Effects of Cholesterol on the Interaction between Liposomes and Ethanol. Biophysical Journal, 78, 2486-2492. https://doi.org/10.1016/S0006-3495(00)76793-2

[14] Arsov, Z. and Quaroni, L. (2007) Direct Interaction between Cholesterol and Phosphatidylcholines in Hydrated Membranes Revealed by ATR-FTIR Spectroscopy. Chemistry and Physics of Lipids, 150, 35-48. https://doi.org/10.1016/j.chemphyslip.2007.06.215

[15] Urbina, J.A., Pekerar, S., Le, H., Patterson, J., Montez, B. and Oldfield, E. (1995) Molecular Order and Dynamics of Phosphatidylcholine Bilayers Membranes in the Presence of Cholesterol, Ergosterol and Lanosterol: A Comparative Study Using ${ }^{2} \mathrm{H}$-, ${ }^{13} \mathrm{C}$ - and ${ }^{31} \mathrm{P}-\mathrm{NMR}$ Spectroscopy. Biochimica et Biophysica Acta, 1238, 163-176. https://doi.org/10.1016/0005-2736(95)00117-L

[16] Meyer, F.J.M., Benjamini, A., Rodgers, J.M., Misteli, Y. and Smit, B. (2010) Molecular Simulation of the DMPC-Cholesterol Phase Diagram. Journal of Physical Chemistry B, 114, 10451-10461. https://doi.org/10.1021/jp103903s

[17] Yoshida, D., Yokoyama, T., Shimoaki, T., Tomita, T., Yoshida, T., Yamamoto, Y., Taga, K., Sumino, A., Dewa, T., Nango, M., Yamamoto, M. and Sheravani, Z. (2013) Morphology Observation of Dipalmitoyl Phosphatidyl Choline (DPPC) Monolayer on Water Surface by Dropping Method. Journal of Biophysical Chemistry, 4, 115-121. https://doi.org/10.4236/jbpc.2013.44016

[18] Yamamoto, M., Suzuki, M., Kimura, T. and Itoh, K. (2008) Molecular Structures at Free Surfaces of Liquid $n$-Heptadecane Investigated by Infrared External Reflection Spectroscopy. The Journal of Physical Chemistry C, 112, 13232-13239. 
https://doi.org/10.1021/jp801414t

[19] Yamamoto, Y., Yokoyama, T., Yoshida, D., Mori, H., Sekiguchi, K., Shimoaki, T., Yoshio, A., Taga, K., Shervani, Z. and Yamamoto, M. (2015) Interactions between Phospholipid Monolayers (DPPC and DMPC) and Anesthetic Isoflurane Observed by Quartz Crystal Oscillator. Journal of Biophysical Chemistry, 6, 42-53. https://doi.org/10.4236/jbpc.2015.62005

[20] Yamamoto, Y. and Taga, K. (2016) Lipid Monolayer and Interaction with Anesthetics. Encyclopedia of Biocolloid and Biointerface Science, 1, 36-58. https://doi.org/10.1002/9781119075691.ch3

[21] Nakagaki, M., Tomita, K. and Handa, T. (1985) Interaction of Differently Oriented Lipids in Monolayer: Mixed Monolayers of 16-(9-Anthroyloxy)Palmitic Acid with Phosphatidylcholine and Cholesterol. Biochemistry, 24, 4619-4624. https://doi.org/10.1021/bi00338a021

[22] Mingotaud, A.F., Mingotaud, C. and Patterson, L.K. (1993) Handbook of Monolayers, Volumes 1 and 2. Academic Press, San Diego, CA.

[23] Janout, V., Turkyilmaz, S., Wang, M., Wang, Y., Manaka, Y. and Regen, S.L. (2010) An Upside down View of Cholesterol's Condensing Effect: Does Surface Occupancy Play a Role? Langmuir, 26, 5316-5318. https://doi.org/10.1021/la100878s

[24] Kim, K., Kim, C. and Byun, Y. (2001) Preparation of a Dipalmitoyl Phosphatidyl choline/Cholesterol Langmuir-Blodgett Monolayer That Suppresses Protein Adsorption. Langmuir, 17, 5066-5070. https://doi.org/10.1021/la0102096

[25] Ohe, C., Sasaki, T., Noi, M., Goto, Y. and Itoh, K. (2007) Sum Frequency Generation Spectroscopic Study of the Condensation Effect of Cholesterol on a Lipid Monolayer. Analytical and Bioanalytical Chemistry, 308, 73-39. https://doi.org/10.1007/s00216-006-1030-0

[26] Chapman, D., Owens, N.F., Phillip, M.C. and Walker, D.A. (1969) Mixed Monolayers of Phospholipids and Cholesterol. Biochimica et Biophysica Acta, 183, 458-465. https://doi.org/10.1016/0005-2736(69)90160-6

[27] Worthman, L.A.D., Nag, K., Davis, P.J. and Keough, K.M. (1997) Cholesterol in Condensed and Fluid Phosphatidylcholine Monolayers Studied by Epifluorescence Microscopy. Biophysical Journal, 72, 2569-2580. https://doi.org/10.1016/S0006-3495(97)78900-8

[28] Yassine, W., Milochau, A., Buchoux, S., Lang, J., Desbat, B. and Oda, R. (2010) Effect of Monolayer Lipid Charges on the Structure and Orientation of Protein VAMP1 at the Air-Water Interface. Biochimica et Biophysica Acta, 1798, 928-937. https://doi.org/10.1016/j.bbamem.2010.01.009

[29] Flach, C.R., Mendelsohn, R., Rerek, M.E. and Moore, D.J. (2000) Biophysical Studies of Model Stratum Corneum Lipid Monolayers by Infrared Reflection-Absorption Spectroscopy and Brewster Angle Microscopy. The Journal of Physical Chemistry B, 104, 2159-2165. https://doi.org/10.1021/jp9936805

[30] Seoane, R., Mi.ones, J., Conde, O., Mi.ones Jr., J., Casas, M. and Iribarnegaray, E. (2000) Thermodynamic and Brewster Angle Microscopy Studies of Fatty Acid/Cholesterol Mixtures at the Air/Water Interface. The Journal of Physical Chemistry B, 104, 7735-7744. https://doi.org/10.1021/jp001133+

[31] Mascetti, J., Castano, S., Cavagnat, D. and Desbat, B. (2008) Organization of $\beta$-Cyclodextrin under Pure Cholesterol, DMPC, or DMPG and Mixed Cholesterol/Phospholipid Monolayers. Langmuir, 24, 9616-9622.

https://doi.org/10.1021/la8004294 
[32] Snyder, R.G. and Strauss, H.L. (1982) C-H Stretching Modes and the Structure of n-Alkyl Chains. 1. Long, Disordered Chains. Journal of Physical Chemistry, 86, 5145-5150. https://doi.org/10.1021/j100223a018

[33] Maroncelli, M., Strauss, H.L. and Snyder, R.G. (1984) The Distribution of Conformational Disorder in the High-Temperature Phases of the Crystalline n-Alkanes. Journal of Chemical Physics, 82, 2811-2824. https://doi.org/10.1063/1.448280 